\title{
Current status and attitudes to self-care training in UK medical schools
}

\author{
Eunice J. Minford ${ }^{1}$ and Christopher L. Manning ${ }^{2^{*}}$
}

\begin{abstract}
Objective: To establish the attitudes towards and current status of self-training for medical students in UK medical schools.

Methods: We emailed a short online questionnaire to the Deans of 34 medical schools in the UK to obtain quantitative and qualitative responses regarding attitudes to self-care training and its current status in each medical school.

Results: A response rate of $44 \%(n=15)$ was achieved. Although $80 \%(n=12)$ of respondents agreed that the quality of care given by a doctor depends on the quality of care they give to themselves, only $67 \%$ agreed that self-responsibility through daily life choices is key to true health and wellbeing. Medical schools were divided as to whether a doctor's first concern should be to care for themselves so that they have a lived quality of care to give to others and $2 / 3^{\text {rd }}$ felt that the GMC requirement for doctors to put the patient's needs first did not lead to doctors subjugating their own needs resulting in burnout and maladaptive coping strategies, yet $73 \%(n=11)$ felt self-care training had the potential to address current rates of burnout. Qualitative responses show there is a need and an appetite for self-care training that needs to be part of the culture of medicine. Some were keen to highlight that toxic systems, environments and culture also need to be addressed.

Conclusions: Despite recognizing the importance and benefits of self-care training and its potential to address current rates of burnout, only $67 \%$ of medical school respondents felt that self-responsibility through daily life choices is the key to health and wellbeing. Medical schools are divided on the impact that always putting patients needs first has on the rates of burnout and maladaptive coping strategies. There is a need for true self-care education for those in charge of medical education in order to understand and appreciate the role of selfresponsibility in personal health and wellbeing and the empowerment it brings to address toxic environments and cultures as well as enhancing the quality of patient care.
\end{abstract}

Keywords: Self-care, Burnout, Responsibility, Student/doctor wellbeing

\section{Background}

The wellbeing of the medical profession is in deep trouble with doctors experiencing unprecedented levels of burnout $[1,2]$ and higher rates of addiction, suicide, anxiety and depression than the general population [3]. These issues are also significantly present amongst medical students [4] and need to be addressed at an early stage by teaching medical students how to truly care for themselves if current trends are to be reversed. Of course, this is not merely for the wellbeing of the

\footnotetext{
* Correspondence: chris.manning@upstreamhealthcare.org

${ }^{2}$ Action for NHS Wellbeing, London, UK

Full list of author information is available at the end of the article
}

students/staff alone, although given the current statistics there is clearly a well-warranted need, but is also required to enhance the safety and quality of care given to patients whilst enabling the NHS workforce at large to model the same healthy behaviours that need to be encouraged amongst the public.

Research has confirmed that patient outcome is influenced and impacted by doctor wellbeing $[5,6]$. And, in essence, a doctor who is self-caring is naturally more able to care for others. We cannot give what we do not ourselves have - so doctors who for whatever reason do not truly care for themselves, are less well equipped to care for others. Self-care is a way of living that cares for 
all dimensions of the self (mental, physical, emotional and spiritual) and encompasses all the thoughts, behaviours, activities, choices and movements that engender that way of life. A student or doctor who lives in a way that is truly self-caring for all dimensions of the self will be reflected in a high level of wellbeing where there is a consistent steadiness \& unflappability, joyfulness, vitality, vibrancy, lightheartedness, compassionate presence and ability to work hard without being detrimentally affected by that work, void of mal-adaptive coping strategies (e.g. drinking alcohol, taking drugs, over-eating, overexercising etc.)

Medical education and the culture of medicine place high demands on medical students yet they do not as yet equip them with the skills to meet those high demands in a way that is truly healthy as evidenced by current rates of suicide, addiction and burnout [1-3]. The culture of medicine and medical training can be described as 'toxic' due to the consequences on student/ doctor wellbeing of high academic demands along with bullying, undermining, harassment, discrimination, abuse and chronic criticism [7]. Novack et al [8] suggest that the development of self-awareness, personal growth and well-being among medical students represent fundamental requirements in the development of 'physicianhealers' who are able to provide whole-person care for their patients as a consequence of living it for themselves.

Furthermore, given the ever-increasing recognition of the role of lifestyle in many conditions there is a growing awareness of the need for personal responsibility when it comes to health and wellbeing. Steve Boorman stated in 2009 that: "What we have got to do is raise awareness that your own individual health is your own responsibility. We are calling for basic training to include that even for surgeons." [5, 9] Furthermore, he said that this "requires nothing less than a sea change in the way in which staff health and wellbeing are perceived" $[5,9]$.

We were informally aware that some medical schools had implemented some form of self-care training but did not know the extent to which this service was provided across the UK medical schools. Given this background, we wanted to evaluate the current attitudes towards self-care training in UK medical schools and to establish the extent to which self-care training is currently being implemented.

\section{Methods}

The contact details for medical school Deans were obtained from the Medical Schools Council website and online Google search between October and November 2015. A short online survey monkey questionnaire was designed to explore the attitudes to and understanding of the role of self-care in medical education as well current rates of implementation. It was sent by email to each medical school Dean in December 2015 with a request for the questionnaire to be completed by them or a medical education professional nominated by them. It was assumed that the Dean or the nominated representative were conveying the attitudes of the Medical School towards self-care. A follow up email was sent in January 2016 with a further request to complete the questionnaire. The questionnaire was not piloted as the pilot participants would also be part of the study group. However, it was reviewed to ensure readability and comprehension. The questionnaire was designed to yield quantitative and qualitative results with areas for free text responses.

\section{Results}

After one email the response rate was $23.5 \%(n=8)$ and this rose to $44 \%(n=15)$ after the second email.

$80 \%(n=12)$ of respondent medical schools agree or strongly agree that the quality of care given by a doctor depends on the quality of care that a doctor gives to themselves. However, only $67 \%$ agree that selfresponsibility through daily life choices is the key to true health and wellbeing. $77 \%(n=11)$ agree or strongly agree that self-care training is needed so that doctors and students can inspire and advise patients to self-care, with $87 \%(n=13)$ agreeing or strongly agreeing that it is needed to improve student/doctor health and wellbeing. Yet only $60 \%$ feel it should be a mandatory part of medical education and only $20 \%$ feel there should be implementation of a nationally agreed curriculum on self-care.

$67 \%(n=10)$ of medical schools did not agree that the GMC requirement for doctors to make the care of the patient their first concern lead to doctors subjugating or being unaware of their own needs, resulting in maladaptive coping strategies or burnout, with $n=10$ scoring 5 or less and $n=5$ scoring 6 or more on a scale of 1 to 10 where $1=$ completely disagrees and $10=$ completely agrees, with overall weighted average score of 4.13.

The proposition that a doctor's first concern should be to care for themselves so that they have a lived quality of care to give to others yielded a range of responses from completely disagree to completely agree, with $53 \%(n=$ $8)$ scoring 5 or less and $47 \%(n=7)$ scoring 6 or more with weighted average of 5.53 .

$73 \%(n=11)$ scored 6 or more to agree that prioritising self-care has the potential to turn around current rates of burnout by establishing a workforce who know how to work hard, play healthy and not get detrimentally affected by the work they do, with $20 \%$ agreeing completely. $27 \%(n=4)$ scored 5 or less and the overall weighted average is 6.93 .

Self-care training is currently mandatory for all students at $40 \%$ of respondent medical schools and is 
available to some extent in $93 \%$ and can include student led initiatives. Between 1 and 10\% of medical students receive self-care training through student selected modules at $61 \%(n=8)$ of medical schools and in one school $100 \%$ of medical students receive self-care training through student selected modules. $77 \%$ of medical schools devoted $1-5 \%$ of the curriculum to self-care training for all students but it was acknowledged that this was difficult to quantify.

Given the competition for space in the curriculum for self-care we wanted to gauge the current climate with respect to the medical curriculum regarding what should go, and what should be consolidated or introduced. There was a desire to see the end of didactic professionalism teaching along with the culture that makes students think disclosing concerns of stress or wellbeing issues will lead to fitness to practice investigations. There was also a desire to rationalize the curriculum and reduce the excessive burden of assessments, providing more time for students to look after themselves.

Areas that the medical schools wished to see consolidated further were mindfulness training for all, talks by Doctors who have been sick, resilience training and relationship to patient safety, alcohol and drug misuse training, teaching on personal fitness, wellbeing and positive mental health, relationships with counsellors and their involvement in developing self-care strategies, values training and self-awareness, student support services, sessions on student stress and healthy doctor-healthy patient. It was acknowledged that multiple strategies are required and one of the key areas that needs addressing is how to achieve student engagement with self-care/ wellbeing as even when it is part of the core curriculum not all attend or see the importance of it.

Topics that the medical schools would like to see introduced are values based practice, more medical humanities and self-care, resilience and wellbeing training throughout the curriculum. In addition, self-care training for all the staff who deliver the program in recognition of the importance of having people who are engaged with the subject and who apply it in their own lives, with more resources for personal and academic tutors to enable academic and pastoral support that build students capability in all areas.

A free text box space was included for medical schools to make any additional comments regarding self-care training. There was a concern that self-care training could be turned into another 'to do' exercise that added extra burden to the students and the issue of getting student engagement was highlighted. It was also expressed that self-care training should be part of the culture of medicine and 'be evident throughout every element of the course, present in every teacher and spoken about amongst peers. We need a culture change where students come to self-care through their own choice rather than have it forced upon them in order to make them 'better' doctors. Self-care should be strategies that can help students feel more at ease with themselves in the present, for themselves and their own wellbeing and pleasure in life'. This was also exemplified in the comment that, 'I do feel the way a student is treated by medical school will reflect their style of care'.

Three respondents were keen to emphasize that selfcare training alone is not the only answer and that there needed to be change to the toxic systems and environments in which doctors work 'that create the situations of bullying, pressure, and overwork that cause stress.' One also expressed concern that by the tenor of our questions the responsibility for maintaining health and wellbeing was 'being squarely laid at the feet of medical students and doctors but they often have to work in very unhealthy environments - both physically and mentally - perhaps those problems should be addressed first.'

\section{Discussion}

There is clearly an appetite for and recognition of the need for self-care training to improve student/doctor health and wellbeing as well as being able to inspire patients to self-care. The majority of medical schools who responded to this survey have implemented some form of self-care training, albeit widely disparate in nature and in nearly all cases only available to $1-10 \%$ of students through student selected modules. Whilst recognising the potential for self-care training to turn around the current rates of burnout in the profession and to enhance personal health and wellbeing it is perhaps surprising that only $67 \%$ of respondent medical schools agreed that self-responsibility through daily life choices is the key to true health and wellbeing. The influence of lifestyle on health and wellbeing is an area that just keeps expanding with more and more conditions being added to the list [10-12]. The major causes of death in the western world through non-communicable diseases like cardiovascular disease, stroke, diabetes, chronic lung disease and cancer are mostly related to lifestyle factors [13] - showing that we are more responsible for the state of our health and wellbeing than we have previously acknowledged or considered or perhaps even wish to consider as we may not want or feel able to give up or change our ways of living. Indeed, it is often only when illness and disease strike that people will reconsider and change their lifestyle but perhaps it would make more sense to do this before we get sick? If the doctors of tomorrow are to inspire their patients to selfcare and adjust their lifestyle, then the doctors of today need to be living that themselves so it is taught and reflected to medical students. This was acknowledged by the desire to bring in training for staff who are to teach 
the students in self-care and to have people who live and apply self-care in their own lives and don't just regurgitate knowledge regarding what is considered to be healthy. This is crucially important as self-care is, unlike many subjects taught at medical school, not reliant on knowledge acquisition, information and recall. It is very much a lived science and only someone who lives it can present it with the true authority and power of the lived experience and knowing of what is truly self-caring and what is not. As a simple example, there is no integrity in a teacher who drinks alcohol advising students not to drink alcohol as it does not come from a body that lives and knows the benefits of such a choice. What is offered in this paper regarding self-care comes from a body (EM) that has lived both a self-destructive way of life and a self-caring way of life and thus it is not mere opinion but lived experience and embodied knowing of what is truly self-caring and what is not, combined with a deep philosophical understanding that is lived and applied. Everyone is free to accept what is offered for consideration or to reject it as they wish.

Self-care training is not just important for the wellbeing of the students and future doctors but also so that they have the skills to be able to advise their patients of the same and become true role models for health and wellbeing. Given the vast number of NHS consultations on a daily basis, a self-caring medical profession has a great opportunity to inspire patients to do likewise and to improve patient outcomes [5].

Steve Boorman has stated that our 'own individual health is our own responsibility' and that this should be taught to medical students and trainees including surgeons $[5,9]$. The more we take and accept personal responsibility for health and wellbeing, the more empowered we are as we realise we have the power to live differently, to make different choices and thus not be a victim of things happening to us over which we have no control. The latter is a feature of the biomedical model but as we shift from the biomedical to the biopsychosocial and to the holistic model of illness and disease manifestation, the level of personal responsibility increases and so too does the level of empowerment. We come to realise that our daily life choices which constitute our lifestyle, are a form of medicine and can be good medicine or bad medicine depending on the choices we make. Our bodies experience all that we live and are impacted by our choices and the more we accept and live this the more power we have over our own health and wellbeing. This is being confirmed by the research showing the role of lifestyle on illness and disease manifestation [10-13]. Likewise it follows that making different lifestyle choices can contribute to health and wellbeing [14] and can be borne out by personal experience and experimentation with those choices.
Hence, the emphasis on personal responsibility for health and wellbeing is intended and is important as it is the key to true health and wellbeing - a fact that as human beings we don't like to hear as it is always easier to blame something outside of ourselves. This may account for some of the contradictory results that place an emphasis on personal responsibility for if agreed to it then asks us to consider to what degree we are taking responsibility for our health through our daily choices and consider changing them, whereas disagreeing with it allows us to continue as we are in the belief that other factors may be more important than the way we live every day. Therefore, whilst we fully acknowledge and definitely agree with the need to address toxic systems and environments as part of creating safe, nurturing and healthy workplaces [15], our ability as human beings to both recognise such toxic systems and to address them in an effective way is increased and facilitated when we are more caring for ourselves as we are more aware, present and willing to call out that which is abusive, bullying, harassing or harmful. In addition, even in the most supportive environment a doctor who is not self-caring will be compromising their own health and the quality of care they can give to another.

Self-care training is NOT about producing doctors who tolerate abusive and bullying practices - it is the opposite. It provides them with the skills to both be able to recognise what is abusive and toxic and how to call it out and address it directly. Toxic systems are not stand alone entities - they are systems created by people but where the systems have not been created for people. The people who create or perpetuate the toxic system themselves are not self-caring, for if they were they would not be able to behave in such a way or to create systems that were devoid of true care for people. Toxic systems prioritise quantity over quality; targets, numbers, and throughputs at the expense of the quality of the service and of the employees delivering those outcomes and their quality of health and wellbeing.

Thus whilst both personal and systemic factors need to be addressed, the latter will only be truly addressed when the former is, such that we engender people coming through the system who will be able to change the systems, change the culture, by reflecting a different way and bringing that to all areas of their work.

Despite the support for self-care training only $60 \%$ felt it should be a mandatory part of medical education. This is a surprisingly low percentage given that most recognise the benefits of self-care training both for the wellbeing of the trainee, their future role as doctor and adviser to patients regarding self-care choices and its potential to prevent or turn around rates of burnout. A medical trainee is an expensive commodity and it seems somewhat ludicrous to spend hundreds of thousands 
training doctors [16] only to have them end up leaving the profession or having significant periods of absence or under performance and presenteeism $[17,18]$ due to burnout or maladaptive coping strategies.

Whilst the medical curriculum is always under pressure with many people wanting a slice of the pie for their subject, we feel it is imperative that self-care training is a standard part of every medical curriculum. It could be argued that due to the onerous and demanding nature of a medical career that self-care training is even more important to enhance personal awareness and provide skills that enable a doctor to work hard, play healthy and not get so detrimentally affected by the work they do.

One of the weaknesses of this survey is that we did not go into more depth and detail regarding the exact nature of the self-care training strategies that are offered across the different medical schools. So whilst $93 \%$ offer some form of training we do not know if that is one or multiple tutorials, workshops, courses or lectures and in the majority of schools it is only available to $1-10 \%$ students. It is clear that there is a wide range of strategies available and that each school is in charge of the range of approaches it can offer. Whilst this allows autonomy and making best use of local resources it also means there is no uniformity or consistency in the type of selfcare training that is being offered, making it more difficult to assess the long term impact and outcomes of the different strategies utilized. Whilst we appreciate there is little appetite for a standardized curriculum on this subject and most prefer to establish their own programs guided by local expertise and interests, this could potentially hamper the progress of self-care training if it is not undertaken from a place that understands the whole person and how the different aspects interact and interrelate to know what is truly self-caring and what is not. The body is the key instrument for knowing what is truly self-caring and what is not and self-care training needs to focus on enhancing body awareness and techniques that help people to discern what is truly healthy and what is not by the effects on the body. Simple experiments with daily life choices regarding food, sleep, exercise, emotions etc. can empower people to know through their own body what is healthy and what is not.

We had a good response rate of $44 \%$, aided by sending the follow-up email, an approach that has been affirmed elsewhere as increasing the yield [19]. Bias could occur if those responding were the medical schools with an interest in self-care. Non-responders could occur because the email did not reach the correct person, time constraints/busyness precluding the filling in of the questionnaire, a lack of interest in the subject or a dislike/disagreement with the questionnaire itself.

\section{Conclusions}

In conclusion, although there is evidence of self-care training across the UK medical schools and a recognized need for it, there is a reluctance to appreciate the extent to which we are responsible for our own health and wellbeing and the measures we can take to address both personal and systemic issues. Medicine is a stressful career that demands that those going into it are given the best training to ensure they can cope with the psycho-emotional demands of the job and know what they can do for themselves to reduce the risk of burnout, addiction, suicide and so forth. Our systems of medical education and education in general, currently place more importance on the course and results achieved rather than the human being; an error that needs corrected by placing self-care at the heart of all education systems. We feel this is necessary to effect true change and not just rely on the use of 'quick-fix' modalities that only provide a temporary solution. A toxic system will only be properly addressed when people in charge of the systems are themselves self-caring and create systems that prioritise the care of all people first and foremost - that means staff as well as patients.

Currently the requirement for doctors to put the care of the patient before themselves is flawed and leads to doctors who subjugate their own needs in order to meet the demands of the job and the needs of the patients, leading to burnout and maladaptive coping strategies. That medical educators do not recognize this fact is a concern and perpetuates the harming culture that leads to doctors subjugating their own needs. Rigid adherence to this belief may also account for some of the contradictory results where there is support for self-care on the one hand and a desire to uphold this belief on the other. The quality of care given to patients will only be improved when the doctors caring for them have a lived quality of care themselves such that they are more present, compassionate, understanding, vital, alert, aware, vibrant, joyful, intuitive and able to sense more readily what is going on for the patient and able to respond appropriately in all or most situations rather than react or become anxious and stressed.

It is a myth that needs to be busted that putting others before ourselves provides high quality care - it is instead one of the root ills at the heart of the current medical crisis. It is a myth that is perpetuated through certain religious teachings and cultural beliefs that we should care for others before ourselves. It is the absence of knowing how to truly care for ourselves that results in burned out, addicted and suicidal doctors and a profession that is willing to submit to toxic organizational demands and self- 
abusive ways of living in the mis-belief it provides better care for patients. The more doctors care for themselves, the more they are able to care for others and in reality this is an ongoing relationship that is built and established simultaneously and ongoingly for there is no end to the depth of self-care that can be lived and thus reflected to others.

There is a need for true self-care education for those in charge of medical education in order to understand and appreciate the role of self-responsibility in personal health and wellbeing and the empowerment it brings to address toxic environments and cultures as well as enhancing the quality of patient care.

\section{Abbreviations}

GMC: General Medical Council; NHS: National Health Service; UK: United Kingdom

\section{Acknowledgements}

Not applicable.

\section{Funding}

Not applicable, no funding sources.

\section{Availability of data and materials}

Please contact author for data requests.

\section{Authors' contributions}

Both EM and CM designed the survey, EM administered the survey, collated the results and drafted the manuscript, CM reviewed the results and the manuscript. Both authors read and approved final manuscript.

\section{Competing interests}

The authors declare that they have no competing interests.

\section{Consent for publication}

Not applicable.

\section{Ethics approval and consent to participate}

Ethical approval was not sought as this was an evaluation survey that did not involve any human personal data/information etc. and was concerned only with non-human data in the form of programmes. Consent was given by email response.

\section{Author details}

${ }^{1}$ Northern Health and Social Care Trust, Antrim, Northern Ireland. ${ }^{2}$ Action for NHS Wellbeing, London, UK.

Received: 29 August 2016 Accepted: 3 February 2017

Published online: 08 February 2017

\section{References}

1. Davies M. (2013). Revealed: Half of GPs at high risk of burnout. Pulse. www. pulsetoday.co.uk/your-practice/battling-burnout/revealed-half-of-gps-athigh-risk-ofburnout/20003157.article\#.VOilvULaE70. Accessed 30 Jan 2016

2. Balch C, Freischlag JA, Tait D, Shanafelt MD. Stress and Burnout among surgeons: Understanding and Managing the Syndrome and Avoiding the Adverse Consequences. JAMA Surgery. 2009;144(4):371-6.

3. Beyond Blue: National Mental Health Survey of Doctors and Medical Students. Oct 2013. http://www.beyondblue.org.au/docs/default-source/ research-project-files/bl1132-report-nmhdmss-full-report_web. Accessed 2 Feb 2016.

4. Dyrbye LN, Thomas MR, Shanafelt TD. Systematic Review of Depression, Anxiety and Other Indicators of Psychological Distress Among U.S. and Canadian Medical Students. Acad Med. 2006;81:354-73.

5. Boorman S. The NHS Health and Wellbeing Review. Dept of Health. Nov 2009. http://webarchive.nationalarchives.gov.uk/20130107105354/http:// www.dh.gov.uk/prod_consum_dh/groups/dh_digitalassets/documents/ digitalasset/dh_108907.pdf. Accessed 3 Feb 2016.

6. Paparella G. Understanding staff wellbeing, its impact on patient experience and healthcare quality. Oxford: Picker Institute Europe; June 2015. http:// www.pickereurope.org/wp-content/uploads/2015/06/2015-06-10StaffWellbeingBriefing.pdf . Accessed 15 Feb 2016.

7. Fnais N, Soobiah C, Hong M, Lillie E, Perrier L, Tashkhandi M, Straus S, Mamdani M, Al-Omran M, Tricco A. Harassment and Discrimination in Medical Training: A Systematic Review and Meta-analysis. Acad Med. 2014; 89(5):817-27.

8. Novack D, Epstein R, Paulsen R. Towards creating physician-healers. Fostering medical students' self-awareness, personal growth and well-being. Acad Med. 1999;74:516-20.

9. Eaton L. Take more responsibility for your own health. NHS staff told BMJ. 2009:339:b3406. http://www.bmj.com/content/339/bmj.b3406?sso

10. Anand P, Kunnumakara AB, Sundarum C, et al. Cancer is a Preventable Disease that requires Major Lifestyle Changes. Pharm Res. 2008;25(9):2097116. http://link.springer.com/article/10.1007/s11095-008-9661-9.

11. Global Burden of Disease Study Collaborators 2013. Global, regional, and national incidence, prevalence, and years lived with disability for 301 acute and chronic diseases and injuries in 188 countries, 1990-2013: a systematic analysis for the Global Burden of Disease Study 2013. Lancet. 2015; 386(9995):743-800.

12. Sharma M, Majumdar PK. Occupational lifestyle diseases: An emerging issue. Indian J Occup Environ Med. 2009;13(3):109-12. doi:10.4103/0019-5278.58912.

13. WHO. Global Status Report on Non-communicable Diseases 2010. Published 2011. http://www.who.int/chp/ncd_global_status_report/en/. Accessed 27 Mar 2016

14. Chiuve S, Rexrode KM, Spiegelman D, Logroscino G, Manson JE, Rimm EB. Primary Prevention of Stroke by Healthy Lifestyle. Circulation. 2008;118:947-54.

15. Panagioti M, Panagopoulou E, Bower P, Lewith G, Kontopantelis E, ChewGraham C, Dawson S, van Marwijk H, Geraghty K, Esmail A. Controlled Interventions to Reduce Burnout in Physicians: A Systematic Review and Meta-analysis. JAMA Intern Med. 2017;177(2):195-205. doi:10.1001/ jamainternmed.2016.7674.

16. BMA. How much does it cost to train a doctor in the United Kingdom? https://webcache.googleusercontent.com/search?q=cache:FMShmjQsSocJ: https://www.bma.org.uk/-/media/Files/Word\%2520files/ News\%2520views\%2520analysis/pressbriefing_cost_of_training_doctors.

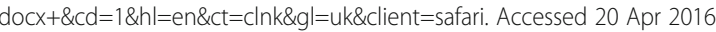

17. Oxtoby K. Why doctors need to resist "presenteeism". BMJ. 2015;351:h6720. doi:10.1136/bmj.h6720

18. Oxtoby K. Why doctors don't take sick leave. BMJ. 2015;351:h6719. doi:10. 1136/bmj.h6719.

19. Cohen L, Manion L, Morrison K. Research Methods in Education, 5th edn. London: Routledge Falmer; 2000. p. 73-90.
Submit your next manuscript to BioMed Central and we will help you at every step:

- We accept pre-submission inquiries

- Our selector tool helps you to find the most relevant journal

- We provide round the clock customer support

- Convenient online submission

- Thorough peer review

- Inclusion in PubMed and all major indexing services

- Maximum visibility for your research

Submit your manuscript at www.biomedcentral.com/submit
Biomed Central 